\title{
Effects of Therapy for Dysphagia in Parkinson's Disease: Systematic Review
}

\author{
Laura W. J. Baijens · Renée Speyer
}

Published online: 18 October 2008

(c) The Author(s) 2008. This article is published with open access at Springerlink.com

\begin{abstract}
This systematic review explores the effects of dysphagia treatment for Parkinson's disease. The review includes rehabilitative, surgical, pharmacologic, and other treatments. Only oropharyngeal dysphagia is selected for this literature search, excluding dysphagia due to esophageal or gastric disorders. The effects of deep brain stimulation on dysphagia are not included. In general, the literature concerning dysphagia treatment in Parkinson's disease is rather limited. Most effect studies show diverse methodologic problems. Multiple case studies and trials are identified by searching biomedical literature databases PubMed and Embase, and by hand-searching reference lists. The conclusions of most studies cannot be compared with one another because of heterogeneous therapy methods and outcome measures. Further research based on randomized controlled trials to determine the effectiveness of different therapies for dysphagia in Parkinson's disease is required.
\end{abstract}

Keywords Systematic review - Dysphagia .

Swallowing disorder - Deglutition · Parkinson's disease · Therapy effect $\cdot$ Therapy outcome $\cdot$ Deglutition disorders

Dysphagia is a frequent symptom of Parkinson's disease [1-3] and is often associated with considerable morbidity and mortality due to nutritional and pulmonary complications $[1,2]$. Dysphagia is observed more often in patients with an advanced stage of Parkinson's disease [4]. In the

L. W. J. Baijens $(\bowtie) \cdot$ R. Speyer

Department of Otorhinolaryngology, Head and Neck Surgery,

University Hospital Maastricht, P.O. Box 5800,

6202 AZ Maastricht, The Netherlands

e-mail: LBA@skno.azm.nl literature, the prevalence of dysphagia varies from 18.5 to $100 \%[2,3]$. However, as many as $15 \%$ of the patients with Parkinson's disease show silent aspiration and do not complain of dysphagia [1]. The pathologic mechanisms of oropharyngeal dysphagia in Parkinson's disease are not quite clear. Oropharyngeal bradykinesia and rigidity, incomplete cricopharyngeal relaxation, reduced cricopharyngeal opening, and delayed initiation of the swallowing reflex have been suggested as possible mechanisms of dysphagia in this patient population [1, 4]. Among the different therapies for dysphagia in Parkinson's disease, rehabilitative treatment (swallowing training), cricopharyngeal sphincterotomy, and pharmacologic therapies have been reported.

The number of reports on the effects of therapies for dysphagia in Parkinson's disease is still rather small. This article is a systematic review of the literature on the effects of treatment for dysphagia in Parkinson's disease. Deane et al. [5] published a systematic review on nonpharmacologic therapies for dysphagia in Parkinson's disease; however, the present systematic review incorporates all therapies available for oropharyngeal dysphagia in Parkinson's disease, including an update on rehabilitative therapies. The different types of therapy are grouped into four main therapy groups: rehabilitative, surgical, pharmacologic, and other.

\section{Methods}

The two authors independently carried out the literature search using the electronic literature databases Embase and PubMed. Differences in their search were settled by discussion. The search was limited to English, German, French, Spanish, and Dutch language publications. In 
Embase the Mesh terms swallowing or dysphagia were combined with Parkinsonism or Parkinson-disease. In PubMed the Mesh terms Parkinson disease or Parkinson disease, secondary or Parkinsonian disorders were combined with deglutition disorders. To identify the most recent publications, the search was supplemented by using free-text words (truncation or wildcard) in Embase and PubMed (for the period after January 2005 up to May 2008): deglut* or swallow* or dysphag* were combined with hypokinetic syndrome or Parkinson* or Paralysis agitans. A total of 602 articles were found in Embase and 258 articles in PubMed. Only articles on the effects of therapy for oropharyngeal dysphagia in Parkinson's disease were included. Studies describing heterogeneous subject populations that included patients with Parkinson's disease and patients with other neurologic diseases were excluded [6], except those studies that presented both pre- and posttreatment data per subgroup [7]. Patients receiving deep brain stimulation were excluded. It was decided to exclude deep brain stimulation because dysphagia has been described as a side effect of this therapy [8]. Studies using a subject population of fewer than three were excluded [911]. The relevance and level of evidence of studies based on populations of fewer than three subjects is doubtful. Percutaneous endoscopic gastrostomy (PEG) tube feeding was excluded as treatment for dysphagia because it is a feeding substitute. There were no limitations on disease duration or disease severity. Articles with subjects of all ages and any duration of treatment were selected. After a first selection based on abstracts, a definitive inclusion was made using the original articles. The reference lists of all the included articles were searched for additional literature. This search did not provide additional studies. In conclusion, 16 articles were included in this review.

\section{Results}

Table 1 presents a summary of the 16 articles that describe rehabilitative, surgical, pharmacologic, or other therapies for dysphagia in Parkinson's disease. The first column of the table represents the level of evidence. To rate the study quality, the ABC rating scale according to Siwek et al. [12] was used. Level A refers to high-quality randomized controlled trials; level $\mathrm{B}$ refers to well-designed, nonrandomized clinical trials, and level $\mathrm{C}$ refers to consensus or expert opinions. Categories A and B were subdivided into two groups according to the way data were handled. The first group used statistical analyses for comparing pre- versus posttherapy data. The second group used descriptive statistics to evaluate the therapy outcome. Level C reflects an expert's opinion without presentation of any statistical data. Authors were listed in alphabetical order. The following data were summarized (if present) for each article: the number of patients, the disease severity based on the Hoehn and Yahr scale (H-Y scale) [13], the kind of therapy used, the evaluation techniques, outcome parameters, blinded rater(s), on/off-motor phase, and the authors' key findings. The fluctuation in daily performance of Parkinson patients on long-term L-dopa therapy is known as the "on/off" phenomenon [14]. The number of subjects refers to the group of subjects on which the study results were based, i.e., dropouts were excluded. All studies were described briefly.

\section{Rehabilitative Treatment (Swallowing Training)}

Studies to determine the effects of swallowing training in Parkinson's disease are extremely rare. El Sharkawi et al. [15] studied the effects of 1 month of Lee Silverman Voice Treatment (LSVT) on swallowing and voice in eight patients with idiopathic Parkinson's disease and dysphagia. The treatment was conducted four times a week at 5060 min per session. The effectiveness of the LSVT was evaluated by comparing pre- and posttherapy measurements, including a lateral videofluoroscopy of swallowing conducted at the same time of day and medicine cycle for each patient. One rater, who was blinded to the therapy used, performed the swallow analysis. Outcome parameters consisted of temporal and qualitative measures of swallowing. Disease severity according to the H-Y scale and statistical analysis were described. After 1 month of LSVT, the authors concluded that LSVT seemed to be effective in improving neuromuscular control of the entire upper aerodigestive tract, improving oral tongue and tongue base function during the oral and pharyngeal phases of swallowing.

In a study by Nagaya et al. [4], the authors tried to determine whether swallowing training, consisting of five motor function/behavioral exercises, improved swallowing function in ten patients with Parkinson's disease and dysphagia. The swallowing training took about $20 \mathrm{~min}$. There was only one session of exercises. The premotor time (PMT) was evaluated pre- and posttherapy during the onmotor phase using electromyography (EMG). The PMT was defined as the latency of EMG activity when a specific movement was requested in response to an imperative stimulus, reflecting the time for information processing in the sensorimotor pathways. The modified barium swallow study (MBS) was performed only before treatment to assess the oral and pharyngeal functions in patients with Parkinson's disease. All examinations were performed during the on-motor phase. Disease severity was mentioned (H-Y scale) and a control population of 12 healthy volunteers was used. It was not mentioned whether the preversus posttreatment EMG results were scored blinded to 


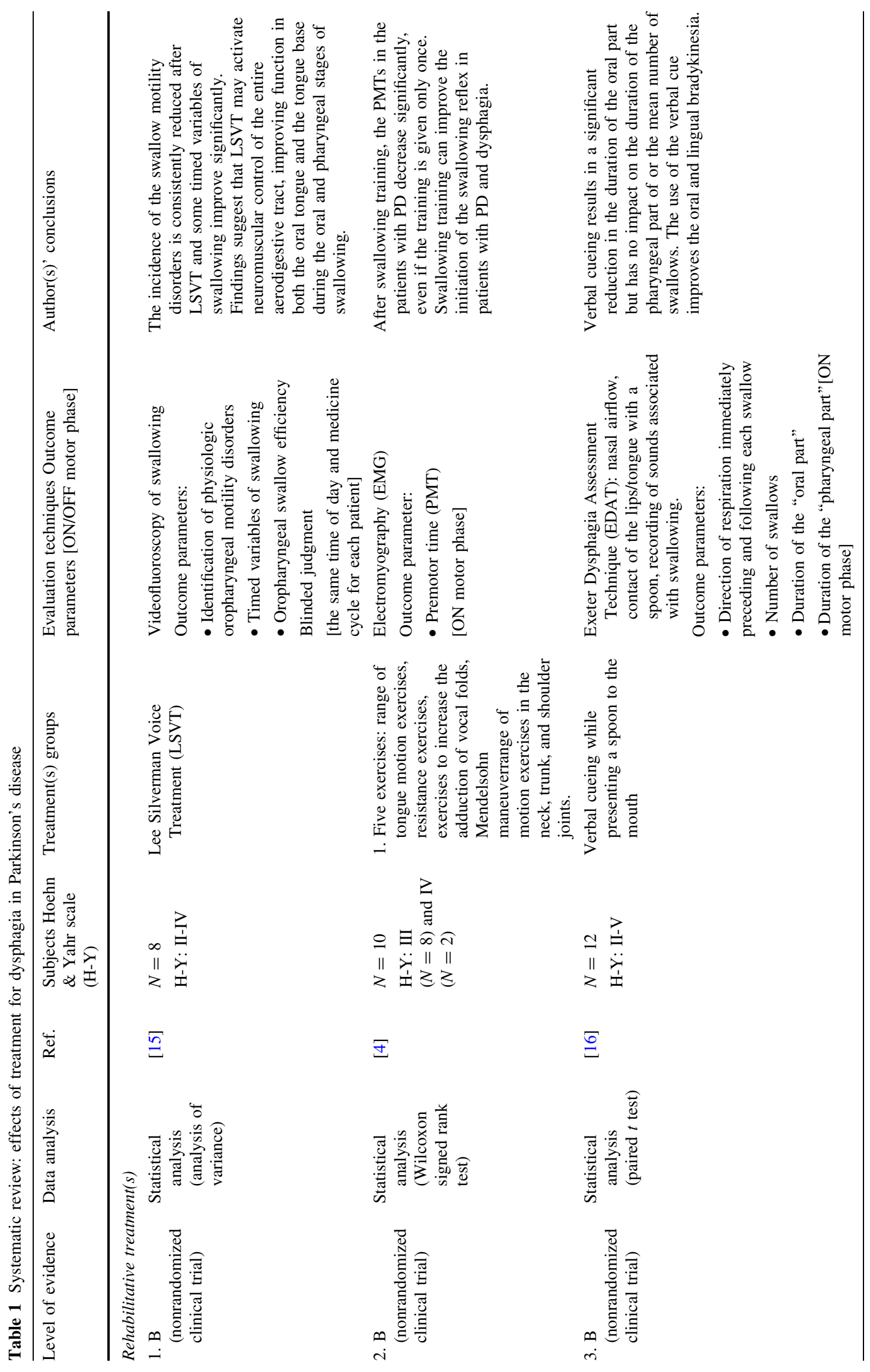




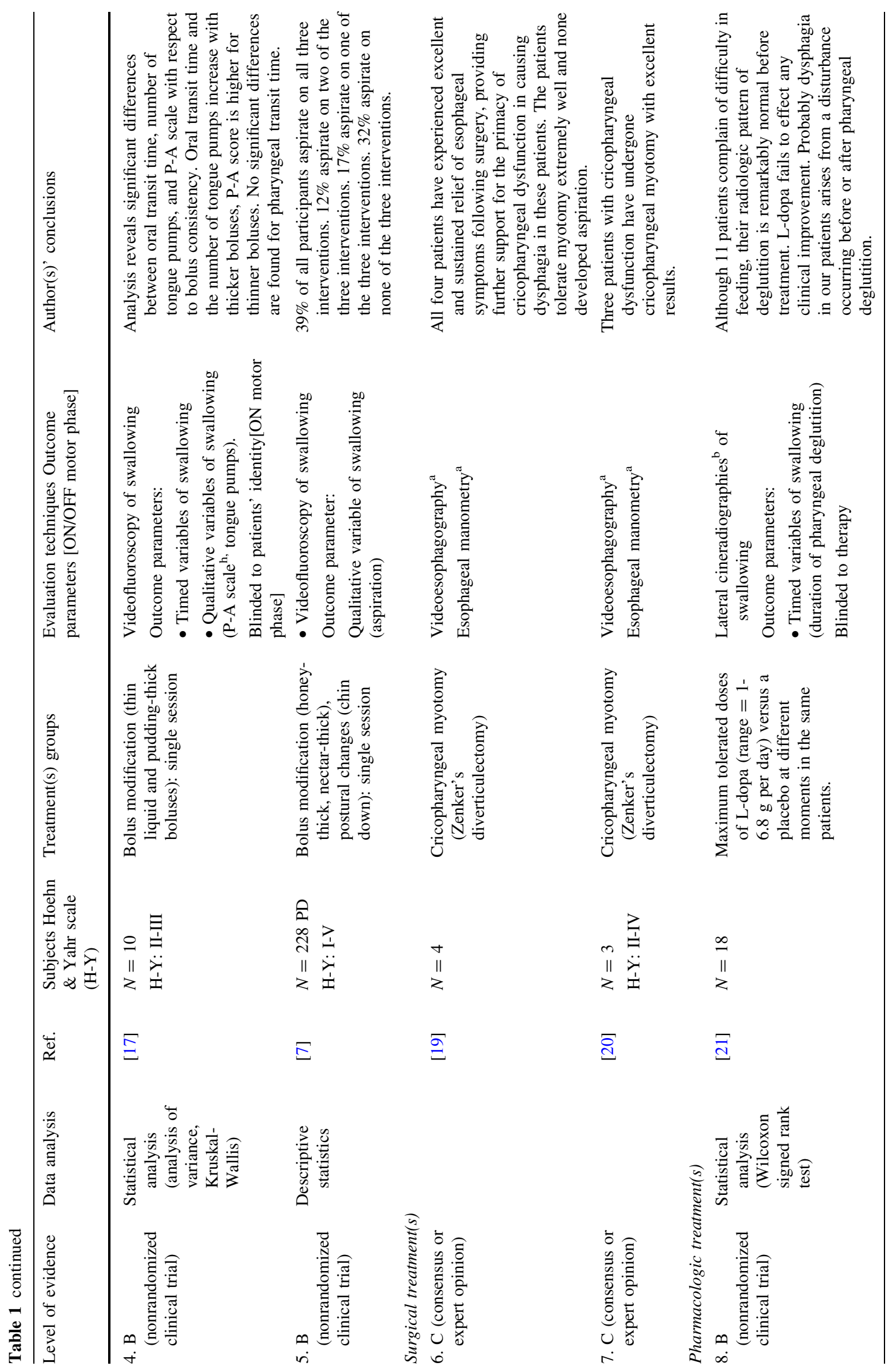




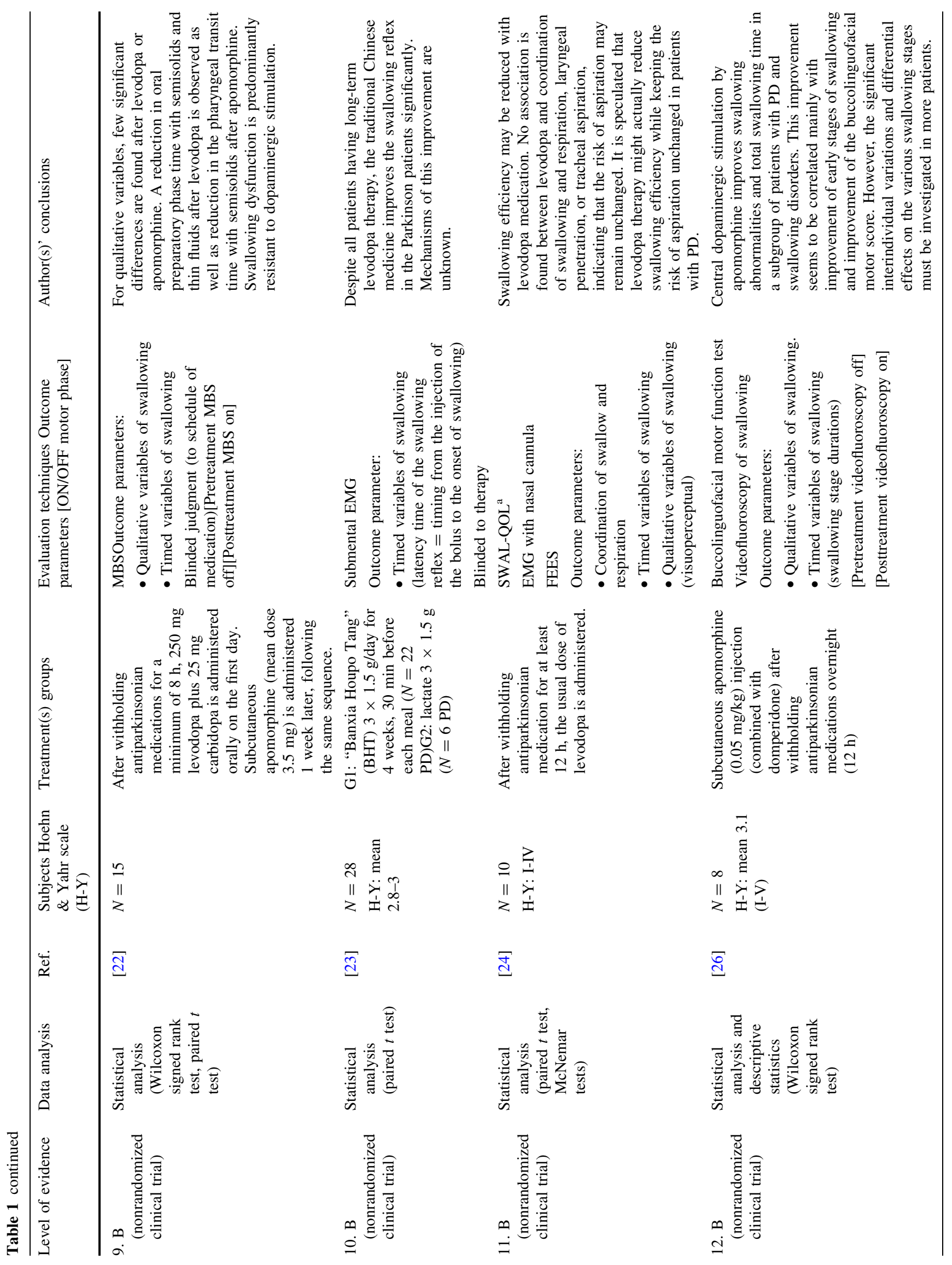




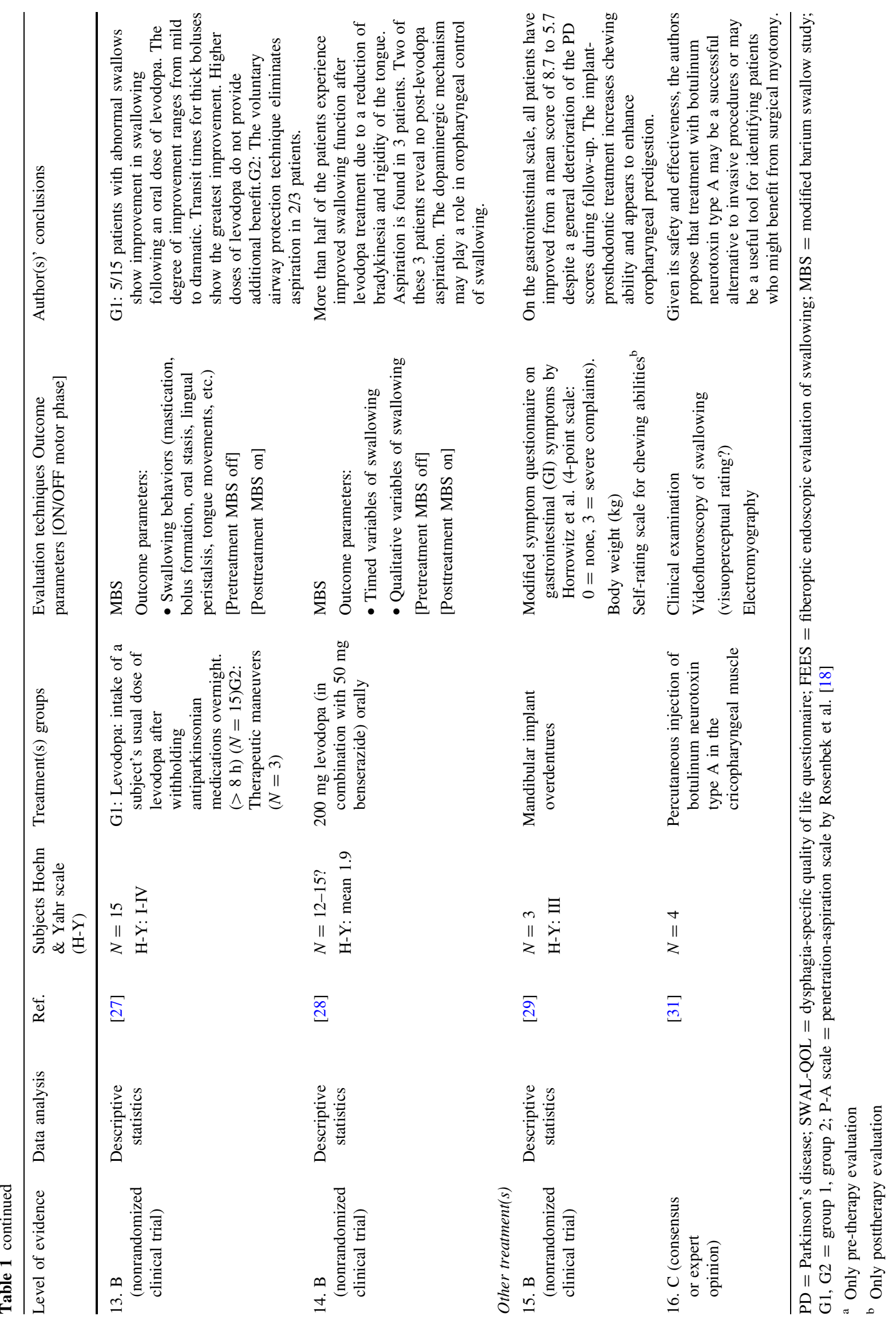


the schedule of treatment. A statistically significant decrease in PMT in patients with Parkinson's disease was observed after swallowing training, even though the training was done only once. The initiation of the swallowing reflex in patients with Parkinson's disease and dysphagia could be improved by swallowing training.

In the report by Pinnington et al. [16], the effects of verbal cueing on the swallowing function while presenting a spoon of liquid to the mouth (on-motor phase) were evaluated by means of the Exeter Dysphagia Assessment Technique (EDAT) in 12 patients with Parkinson's disease compared to 14 control patients on the orthogeriatric ward. The EDAT recorded nasal airflow, contact of the lips or tongue with a spoon, and sounds associated with swallowing. Disease severity was scored according to the H-Y disability scale and the Unified Parkinson's Disease rating scale. It was not mentioned whether the rating during the EDAT was performed blinded to diagnosis and therapy. After statistical analysis the authors concluded that the use of verbal cueing resulted in a significant reduction in the duration of the oral part of swallowing, but had no impact on the duration of the pharyngeal part of swallowing or the mean number of swallows.

Troche et al. [17] evaluated the effect of bolus consistency on timing and safety of swallowing in patients with Parkinson's disease. The severity of the disease was scored using the H-Y scale. During videofluoroscopy, ten Parkinson patients completed a dry swallow, six 5-cc trials of pudding-thick liquid, and six 5-cc trials of thin liquid. The trials were presented in random order. Swallowing was analyzed using qualitative (penetration-aspiration scale [P-A] by Rosenbek et al. [18] and number of tongue pumps) and quantitative (timed variables) swallowing parameters. Patients were tested $1 \mathrm{~h}$ after medication during the on-motor phase. The examiner was blinded to the patients' identity. Analysis revealed significant differences between oral transit time, number of tongue pumps, and penetration-aspiration scale with respect to bolus consistency. Oral transit time and the number of tongue pumps increased with thicker boluses and the P-A score was higher for thinner boluses. No significant differences were found for pharyngeal transit time.

Logemann et al. [7] performed a study to identify which of three treatments for aspiration on thin liquids (chindown posture, nectar-thickened liquids, or honey-thickened liquids) resulted in the most successful immediate elimination of aspiration during a videofluoroscopic swallow study in patients with dementia and/or Parkinson's disease. Two hundred twenty-eight Parkinson patients underwent all three interventions in randomly assigned order. The $\mathrm{H}$ $\mathrm{Y}$ scale was used. The outcome parameter during videofluoroscopy was a qualitative variable (aspiration) of swallowing. No information about the on/off-motor phase during measurements was given. Thirty-nine percent of participants with Parkinson's disease without dementia aspirated on all three interventions. Twelve percent aspirated on two of the three interventions. Seventeen percent aspirated on one of the three interventions. Thirty-two percent aspirated on none of the three interventions. Based on these descriptive results the authors concluded that the most frequently successful intervention to eliminate thinliquid aspiration immediately was the honey-thickened liquid, followed closely by the nectar-thickened liquid and then the chin-down posture.

\section{Surgical Treatment}

Two articles provided anecdotal data about surgical treatment for dysphagia in Parkinson's disease. In the articles by Born et al. [19] and Byrne et al. [20], cricopharyngeal sphincterotomy was performed in very small groups of patients. Born et al. [19] described four patients who experienced excellent and sustained relief of esophageal symptoms following surgery, providing further support for the primacy of cricopharyngeal dysfunction in causing dysphagia in these patients. The patients tolerated myotomy extremely well and none developed aspiration. Byrne et al. [20] studied three patients with cricopharyngeal dysfunction who had undergone cricopharyngeal myotomy with excellent results. Only pretreatment measurements consisting of manometry and videoesophagography were performed in both studies. No posttreatment measurements were described, although the authors' own conclusions were included. In contrast to Born et al. [19], Byrne et al. [20] mentioned disease severity (H-Y scale).

\section{Pharmacologic Treatment}

Seven articles provided information about the effects of antiparkinsonian drugs on the swallowing function.

Calne et al. [21] studied the effect of maximum tolerated doses of L-dopa on swallowing (range $=1-6.8 \mathrm{~g} /$ day) compared to a placebo in 18 patients with idiopathic parkinsonism by means of lateral cineradiography. Data were gathered for both L-dopa and placebo use in the same patients. The outcome parameter was duration of pharyngeal deglutition. Patients and the radiologist were blinded to therapy. Disease severity and on/off-motor phase were not mentioned. Statistical analysis showed no significant difference between the pharyngeal transit times during the intake of L-dopa and of placebo. However, all patients showed remarkably normal radiologic patterns of deglutition before the onset of treatment; only 11 of 18 patients suffered from subjective dysphagia.

Hunter et al. [22] studied the degree of dopaminergic response of swallowing dysfunction in 15 patients with 
Parkinson's disease. After a minimum of $8 \mathrm{~h}$ of withdrawal from antiparkinsonian medication, the patients received a single oral dose of $250 \mathrm{mg}$ of levodopa plus $25 \mathrm{mg}$ of carbidopa on the first study day. The pretreatment MBS was performed during the off-motor phase and the posttreatment MBS during the on-motor phase. One week later the sequence of medication was repeated using subcutaneous apomorphine (mean dose $=3.5 \mathrm{mg}$ ). Outcome parameters consisted of qualitative measures of swallowing function and timed components of swallowing. Two blinded judges performed the rating of the MBS. No information was given about the initial severity of the disease of the included patients. For qualitative variables, few significant differences were found after levodopa or apomorphine. A significant reduction in oral preparatory phase time with semisolids and thin fluids after levodopa was observed, as well as a reduced pharyngeal transit time with semisolids after apomorphine. Using statistical analysis, the authors concluded that swallowing dysfunction was predominantly resistant to dopaminergic stimulation.

The study by Iwasaki et al. [23] described the positive therapy effects of traditional Chinese medicine "Banxia Houpo Tang" (BHT) in improving the swallowing reflex of patients with Parkinson's disease. They studied 28 patients with a defined $\mathrm{H}-\mathrm{Y}$ score and compared them to five healthy controls of comparative age. They divided the patients with Parkinson's disease into two groups. Twentytwo patients and the healthy controls were given BHT $1.5 \mathrm{~g}$ three times a day for 4 weeks at $30 \mathrm{~min}$ before each meal and six patients were given the same amount of lactate. The evaluation tool used was submental electromyography (EMG). Latency time of the swallowing reflex (timing from the injection of the bolus to the onset of swallowing) was used as the outcome parameter. The examiners did not know which patients took BHT or lactate. Whether patients were examined during an on/off-motor phase was not mentioned. The EMG examinations before and after treatment were performed at 11 a.m. After therapy a statistically significant decrease of swallowing reflex time was demonstrated in the patient group receiving BHT. It remains unclear which components in the extract of dried plants were responsible for the improvement of the swallowing function.

Lim et al. [24] studied the effect of the usual dose of levodopa on the coordination of swallowing and respiration in ten patients with Parkinson's disease. The patients were randomly assigned to group 1 or group 2 . Group 1 had the first assessment session during the on-motor phase and the second assessment session during the off-motor phase. Group 2 had the first session during the off-motor phase and the second during the on-motor phase. The two sessions during "on" and "off" were performed in the morning at least 1 week apart. The assessment consisted of EMG with a nasal cannula and an endoscopic evaluation of swallowing (FEES). A dysphagia quality-of-life questionnaire (SWAL-QOL) was filled out only before treatment [25]. Disease severity according to the H-Y scale and statistical analysis were described. No information was given whether the results of the EMG and FEES were scored blinded to therapy. After statistical analysis the authors concluded that swallowing efficiency might be reduced with levodopa medication. No association was found between levodopa and coordination of swallowing and respiration, laryngeal penetration, or tracheal aspiration, indicating that the risk of aspiration may remain unchanged. According to the authors, further studies comprising larger numbers of patients are needed to determine whether the trend in swallowing efficiency is a true effect and to confirm if there really is no difference in risk of aspiration between "on" and "off" states of levodopa.

Tison et al. [26] studied the effects of apomorphine (in combination with domperidone) on buccolinguofacial motoricity and on various swallowing stages by using videofluoroscopy in eight patients with Parkinson's disease and dysphagia. The severity of the disease was scored by the H-Y scale. The pre- and posttherapy assessments included a buccolinguofacial motor function test and videofluoroscopy of swallowing analyzed using qualitative and quantitative swallowing parameters. Only a subjective self-rating of the dysphagia severity on a 10-point scale was carried out pretreatment. Pretreatment measurements were performed during the off-motor phase after overnight withdrawal of medication. Posttreatment measurements were performed during the on-motor phase. It was not mentioned whether rating of the videofluoroscopic examinations was performed blinded to therapy or by how many judges. After statistical analysis the authors found statistically significant results indicating that central dopaminergic stimulation by apomorphine improves swallowing abnormalities and restricts total swallowing time in a subgroup of patients with Parkinson's disease and swallowing disorders. This improvement was correlated mainly with improvement of the early stages of swallowing and improvement of the buccolinguofacial motor score. However, the interindividual variations and differential effects on the various swallowing stages must be investigated in a larger patient population in order to detect statistically significant differences.

In contrast to the previous four articles that used statistical analysis, the following two studies provided descriptive data about the effects of levodopa on the swallowing function.

In the study by Bushmann et al. [27], the effects of the intake of the usual dose of levodopa were evaluated by means of pre- and posttreatment MBS in 20 patients with 
Parkinson's disease. The severity of the disease was scored by the H-Y scale. The number of subjects with Parkinson's disease who were included in the data analysis was not clear. Only 15 of 20 patients had abnormal swallows on MBS before therapy. A second therapy consisting of a voluntary airway protection technique for the mechanical modification of swallowing was applied to three patients. It was not clear whether these three patients also participated in the levodopa trial. An oral motor screening exam and Logemann's bedside dysphagia evaluation were performed only pretherapy. A control group of 13 subjects with no clinical evidence of neurologic or swallowing abnormality was recruited. The pretreatment MBS was performed during the off-motor phase and the posttreatment MBS during the on-motor phase. A variety of swallowing behaviors were assessed during the MBS by two speech pathologists, of which one was blinded to diagnosis. A clear improvement in swallowing in 5 of the 15 patients following an oral dose of levodopa was observed. The voluntary airway protection technique eliminated aspiration in two of three patients. According to the authors, higher doses of levodopa did not provide additional benefit.

Fuh et al. [28] examined the oropharyngeal swallowing ability in 19 Parkinson's disease patients using MBS before and after administering oral levodopa (200 $\mathrm{mg}$ ) in combination with benserazide $(50 \mathrm{mg})$. Salivation and subjective swallowing difficulty were graded on a 5-point scale pretreatment. Salivation was graded as follows: $0=$ normal, $1=$ slight but definite excessive saliva in mouth with possible nighttime drooling, $2=$ moderately excessive saliva with minimal drooling, $3=$ marked excessive saliva with some drooling, $4=$ marked drooling requiring constant use of tissue or handkerchief. Subjective swallowing difficulty was scored on a 5-point scale as well: $0=$ normal, $1=$ rare choking, 2 = occasional choking, 3 = requiring soft food, $4=$ requiring nasogastric or percutaneous endoscopic gastrostomy (PEG) tube feeding. The pretreatment MBS was performed during the off-motor phase and the posttreatment MBS during the on-motor phase. The severity of Parkinson's disease was determined using the H-Y scale and the Schwab and England scores. The judge who rated the radiologic recordings was blinded to the severity of the disease but was not blinded to the time the drugs were taken. Twelve of 19 patients had abnormal swallows on MBS. Six of these 12 patients showed improvement after taking levodopa. Three of 19 patients showed aspiration on MBS. Two of these three revealed no post-levodopa aspiration. It was not clear whether these three patients overlapped with the 12 patients with abnormal swallows. Based on descriptive statistics, the authors concluded that more than half of the patients experienced improved swallowing function after levodopa treatment due to a reduction of bradykinesia and rigidity of the tongue.
Other Treatments

Heckmann et al. [29] studied the benefit of using dental implants combined with overdentures to improve chewing and preingestion capacity in three severely handicapped Parkinson patients. Disease severity was scored according to the H-Y scale. Gastrointestinal (GI) symptoms were assessed pre- and posttreatment using a modified symptom questionnaire described by Horrowitz et al. [30]. Five GI symptoms, i.e., abdominal bloating, heartburn, dysphagia, regurgitation, and constipation, were evaluated on a 4-point severity scale. Body weight was measured before and after therapy. A self-rating scale for chewing abilities was filled out after therapy. It was not described whether the GI symptom score and the self-rating scale for chewing abilities were performed during the on-motor phase. The authors interpreted improved chewing capacity, a moderate gain in body weight, and an improved GI score as signs of improved predigestion.

In the study by Restivo et al. [31], the effect of percutaneous injected botulinum toxin for cricopharyngeal dysfunction was evaluated in four patients with Parkinson's disease by means of videofluoroscopy of swallowing and electromyography. No information was given about the severity of the disease, the on/off-motor phase during measurements, statistics, or whether the rater(s) were blinded to therapy. The method of rating the videofluoroscopy was not described precisely (visuoperceptual?). The authors concluded that given its safety and effectiveness, the treatment with botulinum neurotoxin type A may be a successful alternative to invasive procedures or may be a useful tool for identifying patients who might benefit from surgical myotomy.

\section{Conclusion}

\section{Methodologic Problems}

According to the $\mathrm{ABC}$ rating scale of Siwek et al. [12], none of the included articles were level A (randomized controlled trial). Thirteen of the included articles represented level B (nonrandomized clinical trial); however, some of these studies included very small subject populations (e.g., Heckmann et al. [29]). Three articles represented level $\mathrm{C}$ (expert's opinion). Of the $\mathrm{ABC}$ rating scale, level C (expert's opinion/consensus) provides the least evidence for key clinical recommendations. The included studies on the effects of dysphagia treatment in patients with Parkinson's disease varied in quality of design and most of the included studies had at least one methodologic shortcoming that may have introduced bias. In two articles the therapy effects were evaluated positively by the 
authors despite the fact that only pretreatment examinations were performed $[19,20]$. These articles provided no evidence of efficacy because of the missing posttherapy measurements.

Some studies did not have an adequate method of randomization for referral to different intervention groups, e.g., Iwasaki et al. [23] and Bushman et al. [27] did not score pre- versus posttreatment blinded for the schedule of treatment [4, 24, 26-28], nor did they have an adequate placebo or control population [7, 15, 17, 22, 26-29]. Very often a control group receiving no therapy for dysphagia when in fact an indication for treatment is present is regarded as unethical and therefore usually not approved by any medical ethical committee. Repeated baseline measurements during the waiting period between the referral for therapy and the actual therapy onset could compensate this shortcoming more or less.

Selection bias might be a methodologic shortcoming in some studies. Despite the fact that the control group had neither a history of dysphagia nor evidence of neurologic or respiratory disease, the use of a rather aged subject population (such as the mean subject's age of 78 years in Pinnington's study [16]) makes the presence of dysphagia without subjective complaints more likely. Calne et al. [21] described the effect of maximum tolerated doses of L-dopa on the duration of the pharyngeal phase as outcome. However, pretherapy the pharyngeal phase showed normal radiologic patterns. Now it seems as if L-dopa failed to have an effect in an already normal radiologic pattern of deglutition, whereas the information of the total swallowing act was incomplete. In some articles no information was given about the Parkinson's disease severity scale [19, 21, 22, 31]. Some authors did not describe the "on" or "off"-motor phase during the examinations and therapy [7, 19-21, 23, 29, 31].

Many studies were unbalanced as a result of small sample sizes. Only seven of the 16 studies described in this review included more than ten subjects (maximum $N=228$ ). Four studies used less than five subjects. Such a small number of subjects restricts the possibility to find statistically significant therapy effects. Furthermore, the justification of the generalization of study outcomes to patients with Parkinson's disease in general or to any other patient population with dysphagia can be questioned.

As far as the evaluation tools used to evaluate the therapy effects on dysphagia, the majority of the studies focused on the results found using just one tool $[4,7,15$, $17,21-23,27,28]$. As a consequence, certain aspects of the swallowing act may be missed, such as decreased sensory components of swallowing only detectable during FEES(ST) or a patient's subjective evaluation as measured by a quality-of-life questionnaire. In the literature, diverse evaluation instruments have been used to evaluate therapy effects in dysphagia. When using a restricted set of instruments, the information on therapy outcome will be restricted as well. Poorly defined outcome parameters [19$21,31]$ and inadequate statistical analysis were also causes of reduced reliability.

In conclusion, because of the diversity in study designs, dysphagia treatment, evaluation techniques, and patient characteristics as well as many methodologic problems, statistical pooling of data derived from different articles was not possible.

\section{Therapy Effects in Literature}

The lack of sufficient evidence of the efficacy of different treatments for dysphagia in Parkinson's disease did not suggest a lack of effect. Summarizing the literature, it can be concluded that positive group tendencies might be observed, although no generalized conclusions can be drawn from these reports.

The literature on the effects of surgical therapy for dysphagia is extremely limited. The outcomes of the studies of the two included articles were described in general positive statements by the authors. These statements were not supported by any statistical proof. The five studies on the rehabilitative treatments for dysphagia provided positive tendencies. It would be interesting to design such studies using large subject populations. The seven articles on pharmacologic treatments had very diverse types and doses of medication. Also, their study designs were too diverse to perform statistical pooling of data. Because of different methodologic problems, large randomized controlled trials on pharmacologic treatment for dysphagia were advised. The therapy effects of other treatments such as implant-prosthodontic treatment and percutaneous injection of botulinum neurotoxin type $\mathrm{A}$ in the cricopharyngeal muscle on dysphagia were rather interesting options in the treatment of dysphagia. However, in this field too large well-designed trials are required.

\section{Future Research}

Recommendations for conducting future therapy trials on the effects of different types of treatment for swallowing disorders in Parkinson's disease include the use of large numbers of patients, adequate placebo therapy if possible, a follow-up period after therapy, notation if patients are "on" or "off"-motor phase during measurements and treatment, and using outcome scales validated for dysphagia in subgroups. Nonbiased selection of study participants is necessary. An extended assessment that includes several instruments will provide a more complete view of the 
complexity of therapy effects in dysphagia. An assessment of therapy results might include a quality-of-life measurement, an evaluation of the nutrition intake, an oral motor function test, a FEES, and/or videofluoroscopy of swallowing. Such a multidimensional assessment of the swallowing function pre- and posttherapy is advised because of the complementary aspects of different assessment tools. When using visuoperceptual rating of, for example, videofluoroscopic recordings, an expert panel should be used, blinded to group of therapy as well as to pre- or posttherapy conditions.

It can be concluded that large randomized controlled trials with a multidimensional swallowing assessment are necessary to assess the effectiveness of the different types of dysphagia treatments in Parkinson's disease.

Open Access This article is distributed under the terms of the Creative Commons Attribution Noncommercial License which permits any noncommercial use, distribution, and reproduction in any medium, provided the original author(s) and source are credited.

\section{References}

1. Ali GN, Wallace KL, Schwartz R, Decarle DJ, Zagami AS, Cook IJ. Mechanisms of oral-pharyngeal dysphagia in patients with Parkinson's disease. Gastroenterology. 1996;110:383-92. doi: 10.1053/gast.1996.v110.pm8566584.

2. Clarke CE, Gullaksen E, Macdonald S, Lowe F. Referral criteria for speech and language therapy assessment of dysphagia caused by idiopathic Parkinson's disease. Acta Neurol Scand. 1998;97:27-35.

3. Coates C, Bakheit AMO. Dysphagia in Parkinson's disease. Eur Neurol. 1997;38:49-52. doi:10.1159/000112902.

4. Nagaya M, Kachi T, Yamada T. Effect of swallowing training on swallowing disorders in Parkinson's disease. Scand J Rehabil Med. 2000;32:11-5. doi:10.1159/000112902.

5. Deane KHO, Whurr R, Clarke CE, Playford ED, Ben-shlomo Y. Non-pharmacological therapies for dysphagia in Parkinson's disease. Cochrane Database Syst Rev. 2001;1:CD002816. Review.

6. Robbins J, Gensler G, Hind J, Logemann JA, Lindblad AS, Brandt D, et al. Comparison of 2 interventions for liquid aspiration on pneumonia incidence. Ann Intern Med. 2008;148: 509-18.

7. Logemann JA, Gensler G, Robbins J, Lindblad AS, Brandt D, Hind JA, et al. A randomized study of three interventions for aspiration of thin liquids in patients with dementia or Parkinson's disease. J Speech Lang Hear Res. 2008;51:173-83. doi:10.1044/ 1092-4388(2008/013).

8. Burchiel KJ, Anderson VC, Favre J, Hammerstad JP. Comparison of pallidal and subthalamic nucleus deep brain stimulation for advanced Parkinson's disease: results of a randomized, blinded pilot study. Neurosurgery. 1999;45:1375-84. doi:10.1097/00006 123-199912000-00024.

9. Bailbé M, Bataille B, Paquereau J, Lavazais S, Vandermarcq P, Gil R. Improvement in swallowing difficulties treated by subcutaneous apomorphine infusion after deep brain stimulation in Parkinson's disease. Rev Neurol (Paris). 2004;160:352-3 [in French].
10. Fonda D, Schwarz J, Clinnick S. Parkinsonian medication one hour before meals improves symptomatic swallowing: a case study. Dysphagia. 1995;10:165-6. doi:10.1007/BF00260971.

11. Lieberman AN, Horowitz L, Redmond P, Pachter L, Lieberman I, Leibowitz M. Dysphagia in Parkinson's disease. Am J Gastroenterol. 1980;74:157-60.

12. Siwek J, Gourlay ML, Slawson DC, Shaughnessy AF. How to write an evidence-based clinical review article. Am Fam Physician. 2002;65:251-9.

13. Hoehn NM, Yahr MD. Parkinsonism: onset, progression and mortality. Neurology. 1967;17:427-42. doi:10.1159/000137111.

14. Wajsbort J. The "off-on" phenomenon during treatment of Parkinson's disease with levodopa. J Neurol. 1997;215:59-66. doi: 10.1007/BF00312550.

15. El Sharkawi A, Ramig L, Logemann JA, Pauloski BR, Rademaker AW, Smith $\mathrm{CH}$, et al. Swallowing and voice effects of Lee Silverman Voice Treatment (LSVT): a pilot study. J Neurol Neurosurg Psychiatry. 2002;72:31-6. doi:10.1136/jnnp.72.1.31.

16. Pinnington LL, Muhiddin KA, Ellis RE, Playford ED. Noninvasive assessment of swallowing and respiration in Parkinson's disease. J Neurol. 2000;247:773-7. doi:10.1007/s0041500 70091.

17. Troche MS, Sapienza CM, Rosenbek JC. Effects of bolus consistency on timing and safety of swallow in patients with Parkinson's disease. Dysphagia. 2008;23:26-32. doi:10.1007/ s00455-007-9090-7.

18. Rosenbek JC, Robbins JA, Roecker EB, Coyle JL, Wood JL. A penetration-aspiration scale. Dysphagia. 1996;11:93-8. doi: 10.1007/BF00417897.

19. Born LJ, Harned RH, Rikkers LF, Pfeiffer RF, Quigley EM. Cricopharyngeal dysfunction in Parkinson's disease: role in dysphagia and response to myotomy. Mov Disord. 1996;11:53-8. doi:10.1002/mds.870110110.

20. Byrne KG, Pfeiffer R, Quigley EM. Gastrointestinal dysfunction in Parkinson's disease; A report of clinical experience at a single centre. J Clin Gastroenterol. 1994;19:11-6. doi:10.1097/00004 836-199407000-00004.

21. Calne DB, Shaw DG, Spiers AS, Stern GM. Swallowing in Parkinsonism. Br J Radiol. 1970;43:456-7.

22. Hunter PC, Crameri J, Austin S, Woodward MC, Hughes J. Response of parkinsonian swallowing dysfunction to dopaminergic stimulation. J Neurol Neurosurg Psychiatry. 1997;63: 579-83.

23. Iwasaki K, Wang Q, Seki H, Satoh K, Takeda A, Arai H, et al. The effects of the traditional Chinese medicine, "Banxia Houpo Tang (Hange-Koboku To)" on the swallowing reflex in Parkinson's disease. Phytomedicine. 2000;7:259-63.

24. Lim A, Leow L, Huckabee ML, Frampton C, Anderson T. A pilot study of respiration and swallowing integration in Parkinson's disease: "on" and "off" levodopa. Dysphagia. 2008;23:76-81. doi:10.1007/s00455-007-9100-9.

25. McHorney CA. The SWAL-QOL and SWAL-CARE outcomes tool for oropharyngeal dysphagia in adults. III: Documentation of reliability and validity. Dysphagia. 2002;17:97-114. doi: 10.1007/s00455-001-0109-1.

26. Tison F, Wiart L, Guatterie M, Fouillet N, Lozano V, Henry P, et al. Effects of central dopaminergic stimulation by apomorphine on swallowing disorders in Parkinson's disease. Mov Disord. 1996;11:729-32. doi:10.1002/mds.870110622.

27. Bushmann M, Dobmeyer SM, Leeker L, Perlmutter JS. Swallowing abnormalities and their response to treatment in Parkinson's disease. Neurology. 1989;39:1309-14.

28. Fuh JL, Lee RC, Wang SJ, Lin CH, Wang PN, Chiang JH, et al. Swallowing difficulty in Parkinson's disease. Clin Neurol Neurosurg. 1997;99:106-12. doi:10.1016/S0303-8467(97)00606-9. 
29. Heckmann SM, Heckmann JG, Weber HP. Clinical outcomes of three Parkinson's disease patients treated with mandibular implant overdentures. Clin Oral Implants Res. 2000;11:566-71. doi:10.1034/j.1600-0501.2000.011006566.x.

30. Horrowitz M, Harding PE, Chatterton BE, Collins PJ, Shearman DJC. Acute and chronic effects of domperidone on gastric emptying in diabetic autonomic neuropathy. Dig Dis Sci. 1985;30:1-9.
31. Restivo DA, Palmeri A. Botulinum toxin for cricopharyngeal dysfunction in Parkinson's disease. N Engl J Med. 2002;11:11745. doi:10.1056/NEJM200204113461517.

Laura W. J. Baijens MD

Renée Speyer MS, SLP, PhD 